\title{
Un salaire minimum national
}

\section{Avinash Govindjee}

\section{(2) OpenEdition}

\section{Journals}

Édition électronique

URL : https://journals.openedition.org/rdctss/1673

DOI : $10.4000 /$ rdctss. 1673

ISSN : 2262-9815

\section{Éditeur}

Centre de droit comparé du travail et de la sécurité sociale

\section{Édition imprimée}

Date de publication : 1 avril 2019

Pagination : 170-171

ISSN : 2117-4350

\section{Référence électronique}

Avinash Govindjee, «Un salaire minimum national », Revue de droit comparé du travail et de la sécurité sociale [En ligne], 1 | 2019, mis en ligne le 01 novembre 2021, consulté le 13 novembre 2021. URL http://journals.openedition.org/rdctss/1673 ; DOI : https://doi.org/10.4000/rdctss.1673

\section{(c) (i) (9)}

Revue de droit comparé du travail et de la sécurité sociale est mise à disposition selon les termes de la Licence Creative Commons Attribution - Pas d'Utilisation Commerciale - Pas de Modification 4.0 International. 


\title{
AVINASH GOVINDJEE
}

\author{
FACUlté dE DROIT DE L'UNIVERSITÉ NELSON MANDELA
}

\section{UN SALAIRE MINIMUM NATIONAL}

L'Afrique du Sud a récemment adopté une législation établissant un salaire minimum national. A la fin du mois de novembre 2018, le président a approuvé la loi de 2018 sur le salaire minimum national (la loi 9 de 2018) (la « Loi »), qui définit également la composition et les fonctions de la Commission nationale sur le salaire minimum et les questions connexes. L'adoption de cette Loi était motivée par le constat que l'Afrique du Sud restait, bien des années après l'instauration d'un ordre démocratique, l'une des sociétés les plus inégalitaires au monde, en proie à d'énormes disparités de revenus sur le marché du travail national. Cette Loi a pour objet de faire soutenir le développement économique et faire progresser la justice sociale par les moyens suivants: en améliorant les salaires des travailleurs les moins bien rémunérés; en protégeant les travailleurs contre des salaires déraisonnablement bas; en préservant la valeur du salaire minimum national ; par la promotion de la négociation collective ; en soutenant la politique économique. La Loi s'applique à tous les travailleurs et à leurs employeurs, à l'exception des membres des forces de défense nationale sud-africaine, de l'agence de renseignement nationale et des services secrets sud-africains. Elle ne s'applique pas au travail bénévole, un bénévole étant une personne qui travaille pour une autre personne et qui ne reçoit pas ou n'a pas le droit de recevoir de rémunération pour ses services.

\section{I - LE SALAIRE MINIMUM NATIONAL}

Le salaire minimum national correspond à un montant indiqué dans une annexe de la Loi et est ajusté chaque année. Chaque travailleur a droit à un salaire au moins égal au salaire minimum national et chaque employeur doit verser à ses travailleurs un salaire qui ne soit pas inférieur au salaire minimum national. Le paiement du salaire minimum national ne peut être écarté et l'emporte sur toute disposition contraire de tout contrat, convention collective, accord de branche ou loi. Le salaire minimum national constitue, de fait, une condition du contrat du travailleur, sauf dans le cas où le contrat, la convention collective ou la loi prévoit un salaire plus favorable pour le travailleur. La modification unilatérale par l'employeur des salaires, de la durée du travail ou d'autres conditions de travail en lien avec la mise en œuvre du salaire minimum national serait considérée comme une pratique déloyale. Sauf disposition légale contraire, le calcul du salaire aux fins de la Loi est le montant payable en argent pour les heures de travail effectuées normalement. Cela exclut les sommes versées pour permettre à un travailleur de travailler, comme par exemple les indemnités de transport, d'équipement, d'outils, de nourriture ou de logement, sauf clause contraire dans un accord de branche. Les autres exclusions comprennent les paiements en nature, pension et hébergement compris, sauf clause contraire dans un accord de branche, les gratifications, les primes, les pourboires, les cadeaux et tout autre type de paiement prescrit. A l'heure actuelle, le salaire minimum national est fixé à $20 \mathrm{R}$ par heure de travail ordinaire. Malgré ce minimum général, différents arrangements ont été mis en place pour les ouvriers agricoles (18 R de l'heure), les travailleurs domestiques (15 R de I'heure), les travailleurs employés dans le cadre d'un programme élargi de travaux publics (11 $\mathrm{R}$ de l'heure) et les travailleurs ayant conclu un contrat d'apprentissage visé par l'article 17 de la loi de 1998 sur le développement des compétences (loi nº7 de 1998).

\section{II - LA COMMISSION NATIONALE SUR LE SALAIRE MINIMUM}

La Commission nationale sur le salaire minimum (la "Commission »), créée en vertu de la Loi, doit procéder à un réexamen du salaire minimum national dans les 18 mois suivant l'entrée en vigueur de la Loi. La Commission doit faire des recommandations au ministre du travail sur l'ajustement du salaire minimum national. Ces recommandations doivent être axées sur la réduction de la pauvreté et la diminution des écarts de salaires et des inégalités. La Commission est composée de membres nommés par les représentants des entreprises, 
de la société civile et des organisations syndicales, ainsi que de trois experts indépendants connaissant bien le marché du travail et les conditions de travail, ainsi que d'un président nommé par le ministre. Pour établir ses recommandations, la Commission doit tenir compte des facteurs suivants: I'inflation, le coût de la vie et la nécessité de maintenir la valeur du salaire minimum, les niveaux de salaire et les résultats des négociations collectives, le produit intérieur brut, la productivité, la capacité des employeurs à faire fructifier leur entreprise avec succès, l'activité des petites, moyennes ou microentreprises et des nouvelles entreprises,l'impact probable des ajustements recommandés sur l'emploi ou la création d'emplois, et tout autre facteur pertinent. Les fonctions de la Commission consistent notamment à enquêter et à faire un rapport annuel au ministre sur l'impact du salaire minimum national sur l'économie, la négociation collective et la réduction des écarts de revenus, et à mettre ces informations à la disposition du public.

\section{III - REMARQUES FINALES}

L'introduction du salaire minimum national a suscité de nombreux débats dans le pays. L'un des points de désaccord concerne les dispositions de la Loi qui permettent à un employeur ou à une organisation d'employeurs de demander une exemption du paiement du salaire minimum national. Des règlements sont entrés en vigueur pour compléter les dispositions de la Loi à cet égard, offrant aux employeurs la possibilité de démontrer qu'ils ne peuvent pas se permettre de payer les nouveaux minima, suite à des consultations constructives avec les travailleurs et leurs représentants. Les employeurs qui ne se conforment pas aux dispositions de la Loi seront sanctionnés par le ministère du travail, dont les inspecteurs sont également habilités à enquêter sur la conformité des lieux de travail. Les salariés peuvent également s'adresser directement à la Commission de conciliation, de médiation et d'arbitrage du pays. Cette Loi s'inscrit dans l'approche sud-africaine consistant à établir un lien entre les organisations patronales et celles des travailleurs (telles que définies dans la loi de 1994 sur le Conseil du développement économique et du travail (loi n 35 de 1994) (loi NEDALC). Il est important de noter que la Commission comprend également des membres nommés par la " société civile », à savoir les organisations non gouvernementales visées par la loi NEDALC. La Loi introduit et utilise également le concept de «travailleur», qui est défini comme " toute personne qui travaille pour un autre et qui reçoit, ou a le droit de recevoir un paiement pour ce travail, qu'il soit en argent ou en nature. » C'est une notion différente de celle contenue dans la plupart des lois relatives à l'emploi en Afrique du Sud, qui adoptent généralement des définitions relatives au concept de «salarié » (et qui excluent expressément les travailleurs indépendants). L'approche plus large proposée par la loi fait écho à des jugements récents tels que Pretorius and Another $v$ Transnet Pension Fund and others ${ }^{1}$. Dans cette affaire, qui concernait une plainte émanant de retraités, la Cour constitutionnelle a jugé inutilement contraignant d'exiger des requérants qu'ils plaident l'existence d'une relation employeursalarié. La Cour a noté que selon l'article 23 de la Constitution "toute personne " a droit à des pratiques de travail équitables et qu'il s'agit de protéger les personnes contre les pratiques de travail déloyales nées d'une relation employeur-salarié. La Cour a noté que la jurisprudence en matière de travail reconnaissait que les pratiques de travail déloyales prévues par la LRA pouvaient aller au-delà de la cessation de l'emploi et que les tendances actuelles en matière de travail soulignaient la nécessité d'adopter une vision large du droit à une pratique de travail équitable, car les personnes occupant un emploi formel sont de moins en moins nombreuses. Comme l'a souligné la Cour, « de plus en plus de personnes se retrouvent dans la "zone nébuleuse » comme «sous-traitants indépendants » dans un emploi ponctuel soumis à des sociétés multinationales sans visage qui peuvent opérer à partir d'une présence sur le Web. » La Cour a jugé que les faits de l'affaire constituaient un motif suffisant pour ne pas limiter la protection de l'article 23 de la Constitution aux seules personnes ayant un contrat de travail. 